\title{
Model Predictive Control for Ethanol Steam Reformers with Membrane Separation
}

\author{
Maria Serra, Carlos Ocampo-Martinez, Mingming Li and Jordi Llorca \\ Institut de Robòtica i Informàtica Industrial (CSIC - UPC) \\ Universitat Politècnica de Catalunya \\ Llorens i Artigas 4-6, 08028 Barcelona, Spain \\ Institut de Tècniques Energètiques \\ Universitat Politècnica de Catalunya \\ Diagonal 647, Ed. ETSEIB, 08028 Barcelona, Spain
}

\begin{abstract}
This paper focuses on the dynamic modelling and the predictive control of an ethanol steam reformer (ESR) with Pd-Ag membrane separation stage for the generation of pure hydrogen. Hydrogen purity necessary to feed a proton exchange membrane fuel cell (PEMFC) is required. A non-linear dynamic model of the ESR is developed together with a procedure for adjusting the model parameters in order to fit a bank of experimental data of a real ESR system. Static and dynamic analysis of the non-linear ESR model is presented. From this non-linear model, a linear, reduced order and discretised model is derived and a model predictive controller (LMPC) is designed for the ESR system. Control objectives are pure hydrogen flowrate tracking and ethanol inlet minimization. Comparisons between the non-linear and linear models are carried out to determine the control constraints. Finally, simulation results for the implemented LMPC controller are presented and discussed.
\end{abstract}

Ethanol steam reformer, staged-separation membrane reactor, control-oriented model, linear model predictive control

\section{Introduction}

Nowadays hydrogen has emerged as an interesting energy vector which may be used to store energy from renewable sources. Through fuel cells this hydrogen may be converted into electricity when necessary at a high efficiency. For instance, fuel cell vehicles have efficiencies more than two times higher than those of combustion engines. The most mature fuel cell technology, with a wide range of applications, is PEMFC 
[1]. Durability, reliability and efficiency of PEMFC are significantly improved when pure hydrogen is supplied to them.

Due to its physical properties, hydrogen is difficult to transport and store, which makes the techniques of in situ hydrogen production increasingly interesting for many applications. Hydrogen can be obtained from many different sources such as water, ethanol, natural gas or other fossil fuels. Among these sources, ethanol has been chosen for generating hydrogen in the last decades for several advantages: high content of hydrogen, easy portability and storability, renewable nature and low toxicity.

Nowadays, there are three common techniques for hydrogen production from ethanol: steam reforming (SR), partial oxidation (POX) and auto-thermal reforming (ATR). The best of these options is SR because of its lower operating temperature and higher hydrogen yield. However, this technique generates the highest emission of $\mathrm{CO}$, which can poison the anode catalyst of PEMFCs if the quantity is more than 100 parts per million (ppm). If high temperature PEMFC are used, the tolerance to CO is higher, as explained in [2], where the integration of an ethanol steam reformer to a high temperature PEMFC is studied. In any case, hydrogen purification is essential before feeding PEMFCs.

Conventional methods for pure hydrogen generation include water gas shift (WGS), $\mathrm{CO}$ preferential oxidation (COPrOx), and pressure-swing adsorption (PSA), among others. Additionally, membrane separation techniques have already been applied to obtain pure hydrogen from the gas mixture $[3,4,5,6]$. Especially, palladium-based membranes have gained great interest with more than 6000 scientific articles [7] involved since Juenker et al. [8] analysed the use of palladium membranes for hydrogen purification in 1955. The remarkable progress achieved in the field of palladium-based membrane reactors (MRs) is due to their complete hydrogen perm-selectivity with respect to all other gases [7]. Furthermore, only one heating unit is required for both the reformer stage and separation stage. In [9], an exergetic study of an ethanol steam reformer with a palladium-based membrane has been presented and the best operating conditions regarding the exergetic and thermal efficiency have been determined.

The catalyst plays an important role in the ethanol steam reforming (ESR) process, since it can accelerate the reaction rate and improve the performance of the ESR. Cobalt-based catalysts have been considered to be a suitable choice for their low cost, high activity and selectivity $[10,11,12]$, especially for the ESR to produce hydrogen at moderate temperature [11, 13, 14]. Catalysts setup is also a relevant issue. In [15], ESR for hydrogen generation over structured catalysts is described based on different experimental tests.

Dynamic modelling of ESRs with membrane separation is very limited. In [16], the way to obtain a control-oriented model from a dynamic simulation-oriented model of an ESR without membrane separation stage is presented, while [5] presents the model of a staged-separation membrane reactor for steam reforming of methane. Only [17] presents the modelling of the ESR with membrane separation for the generation of pure hydrogen. In the paper, the ESR model is obtained according to the phenomenological kinetic model with power law [18] and experimental data. On the other hand, so far, there have been very few works involved into designing controllers for ESR. In spite of the non-linear ESR nature, linear controllers are designed in two works [19, 20] since they can yield a satisfactory performance if the process is operated close to a nominal 
operating point. Specifically, in [20], a linear model predictive controller (LMPC) is designed for the ESR without membrane separation.

Model predictive control (MPC) is an advanced strategy of process control that has been widely used in industry and chemical processes since the 1980s [21, 22]. The MPC strategy is a set of control methodologies that use a mathematical model of a considered system to obtain control actions through minimizing a cost function related to selected control objectives considering the desired system performance.

MPC has presented obvious advantages over other methods [21]: multi-objectives easy to deal with the multi-variable system, feed-forward control been used in a natural way to compensate measurable disturbances and beneficial for tracking future references. However, the strategy has also its own drawbacks: its tuning, which is not a general theory that can be implemented in a generic way, and the high computational time derived from the number of variables and constraints, mainly in large-scale system. In [20], an LMPC scheme has been presented for an ESR process without membrane separation.

The main contribution of this paper is to obtain a dynamic model of an ESR with membrane separation and to use this model to design a LMPC able to operate the system properly around a nominal operating point.

The reminder of this paper is organized as follows: Section 2 gives a brief description of the ESR system with membrane separation studied in this work. Section 3 firstly presents the non-linear mathematical model of the ESR and secondly presents the control-oriented model for the MPC controller design. Section 4 presents the MPC design for the ESR, including the formulation of the corresponding optimization problem and the LMPC controller configuration with output feedback. Section 5 shows and discusses the main simulation results. Finally, the main conclusions are drawn in Section 6.

\section{System description}

\subsection{Experimental setup}

For the purpose of simplifying the system setup, the process of generating hydrogen via ESR and purifying hydrogen using selective membrane may be adopted in a single reaction and separation module, named Staged-Separation Membrane Reactor (SSMR) [17]. In this paper, the ESR was conducted into a SSMR using a cobalt-based catalyst over cordierite monoliths that were implemented in series into a stainless-steel Pd-Ag membrane stage [23]. A scheme of the SSMR is shown in Figure 1. The SSMR modelled in this work corresponds to a real laboratory system with the following characteristics: the reaction-separation chamber measures $230 \mathrm{~mm}$ high and $22 \mathrm{~mm}$ of outside diameter and has a lower head to allow the exit of the separated streams, which are retentate and permeate streams. A feed evaporation conduit is used to evaporate both ethanol and water before entering the reactor. Five catalytic honeycomb pieces of 2 $\mathrm{cm}$ length each are disposed in series into the reactor followed by a Pd-Ag membrane tube. The catalytic honeycomb pieces are loaded with a total of $1.32 \mathrm{~g}$ of cobalt-based catalyst. The pine-hole free and dead-end membrane tube measures $76 \mathrm{~mm}$ high, 1/8 
inch diameter and a total area of $7.1 \mathrm{~cm}^{2}$. The Pd-Ag active layer is $30 \mu \mathrm{m}$ thick over a porous stainless steel support. The liquid mixture of the ethanol and water are fed directly from the storage tank by a high-performance liquid chromatography (HPLC) pump. The retentate pressure is adjusted and controlled by a manually-operated backpressure regulator and the permeate pressure is maintained at atmospheric pressure.

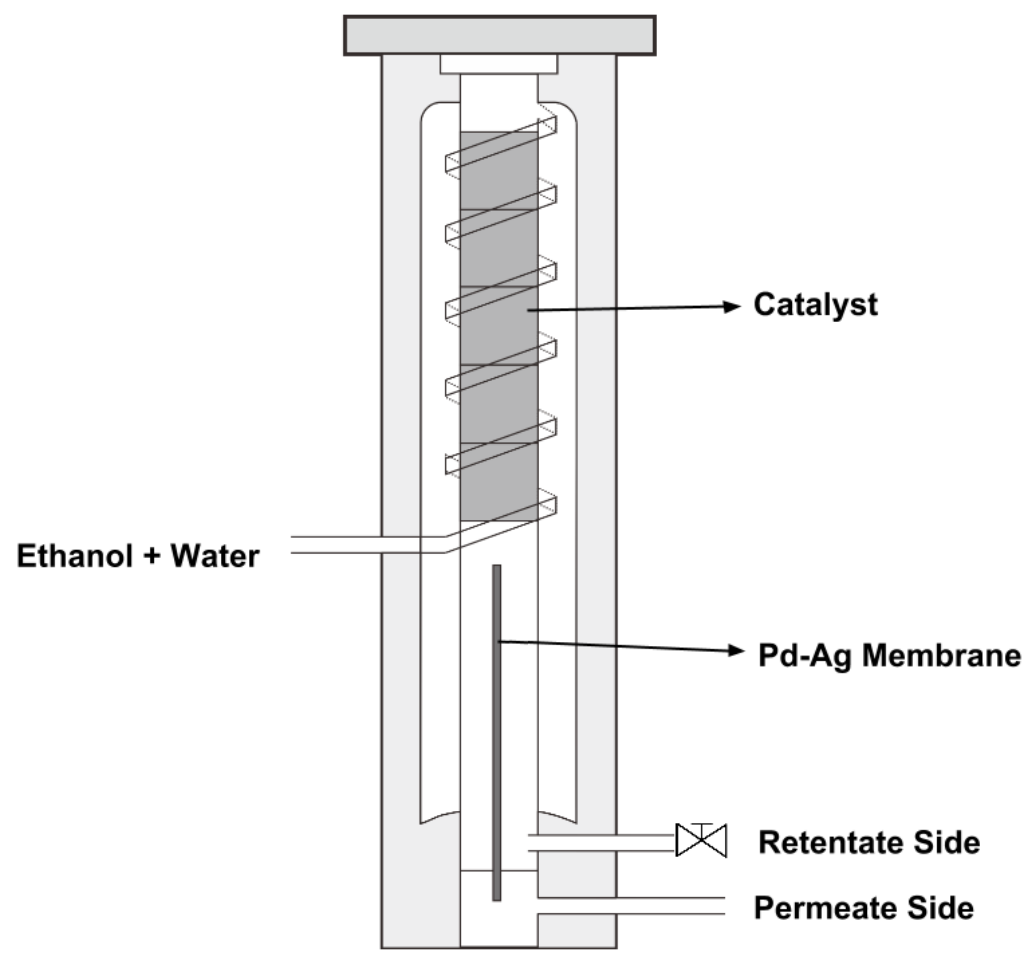

Figure 1: Staged-separation membrane reactor

The experimental data used in this work was obtained at different pressures in the range 1 bar- 14 bar, different temperatures in the range $500^{\circ} \mathrm{C}-600^{\circ} \mathrm{Cand}$ different steam to carbon $(S / C)$ ratios. The steam to carbon ratio is defined as the number of water molecules divided by the number of carbon atoms, and it is applied in this case to the inlet flowrate mixture of both ethanol and water. Also, two sets of experiments were done in order to characterise the behaviour of the system: experiments without the separation stage (without membrane) and experiments with the separation stage (with membrane). A full-conversion rate (100\%) of ethanol and acetaldehyde were measured in the reformer stage. 
Reforming Stage Membrane Separation Stage

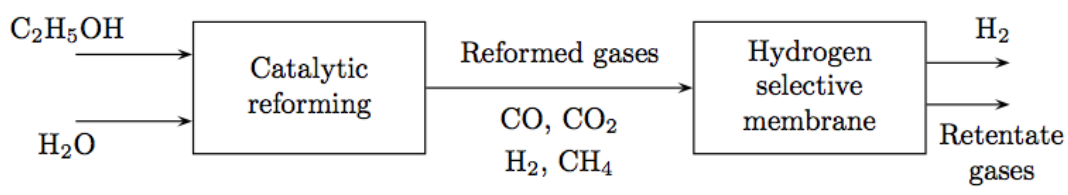

Figure 2: Scheme of the ESR

\subsection{Chemical reaction}

The ESR reactions over cobalt-based catalysts are expressed as follows [12, 24, 25]:

$$
\begin{aligned}
\mathrm{C}_{2} \mathrm{H}_{5} \mathrm{OH} & \longrightarrow \mathrm{CH}_{3} \mathrm{CHO}+\mathrm{H}_{2}, \\
\mathrm{C}_{2} \mathrm{H}_{5} \mathrm{OH} & \longrightarrow \mathrm{CO}+\mathrm{CH}_{4}+\mathrm{H}_{2}, \\
\mathrm{CO}+\mathrm{H}_{2} \mathrm{O} & \longrightarrow \mathrm{CO}_{2}+\mathrm{H}_{2}, \\
\mathrm{CH}_{3} \mathrm{CHO}+3 \mathrm{H}_{2} \mathrm{O} & \longrightarrow 2 \mathrm{CO}_{2}+5 \mathrm{H}_{2} .
\end{aligned}
$$

These four reactions are taking place in the same space and conditions simultaneously. Firstly, ethanol dehydrogenates into hydrogen and acetaldehyde (1a), which is further reformed with water to carbon dioxide (1d). In addition, cobalt catalysts are active for the Water Gas Shift (WGS) reaction (1c) under typical operating conditions. The undesired reaction is the ethanol decomposition to produce carbon monoxide and methane (1b). In the membrane separation stage, the Pd-Ag membrane permeates only the hydrogen and leaves the waste gases in the retentate side [26, 27]. Figure 2 shows a scheme of the process with the two stages in series.

\subsection{Energy study}

An energy study has been done in order to quantify the global energy balance of the SSMR described in Section 2.1 at steady state and for some specific operating conditions. In particular, 8 bar of pressure, $813.15 \mathrm{~K}\left(540 \mathrm{i}_{i} 1 / 2 \mathrm{C}\right)$ of temperature, an inlet water flow of $0.0108 \mathrm{~mol} / \mathrm{min}$ and an inlet ethanol flow of $0.0018 \mathrm{~mol} / \mathrm{min}$ are considered. The relationship between these water and ethanol inlet flows is 6 to 1 , or equivalently $S / C=3$. The energy supplied is calculated taking into account the power necessary to vaporize and heat the reactants to $813.15 \mathrm{~K}$, the energy necessary to pressurize the gases and the heat consumed by the reaction. The energy for the hydrogen pressurisation is not considered. The supplied energy results in 14.78 Watts. On the other hand, for the selected operating point, 3.4 mols of hydrogen are obtained from each mole of ethanol in the reforming stage and therefore, the outlet hydrogen flowrate form the reforming stage is $6.2 \times 10^{-3} \mathrm{~mol} / \mathrm{min}$. This flowrate could be transformed into 29.5 Watts of heat if combusted (High Heating Value used for the calculation). Considering the separation stage as well for the same operating conditions, the hydrogen flowrate in the retentate side is $4.65 \times 10^{-3} \mathrm{~mol} / \mathrm{min}$ and the pure hydrogen flowrate in the permeate side is $1.55 \times 10^{-3} \mathrm{~mol} / \mathrm{min}$. These flowrates could be transformed into 22.15 Watts 
of heat and 3.57 Watts of electricity, respectively, considering that the pure hydrogen feeds a PEM fuel cell with a stoichiometry of 1.1 and 0.8 Volts. This numbers, corresponding to the experimental setup described in Section 2.1, could easily be improved if improved designs were considered.

\section{Mathematical modelling}

\subsection{Main assumptions}

In this paper, two plug-flow kind reactors are assumed to represent the dynamic behavior of the ESR with membrane separation. The first reactor is the reformer stage, and the second is the membrane separation stage. The main modelling assumptions are:

- Isothermal operation conditions within each stage

- Isobaric operation conditions assumed due to the high void fraction of monolithic structures

- Completely mixed fluid inside the plug-flow reactor (PFR) in any cross-section at any position

- Neglected diffusion terms due to the predominance of convection action over diffusion action

- Ideal gases due to the low operating pressure

- Fluid velocity depending only on the axial position

- 1D model due to the small diameter of the reactor

\subsection{Mass balance of the reformer stage}

The mole balance equation together with the initial and boundary conditions of the plug flow reactor model are expressed as [16]

$$
\begin{array}{ll}
\frac{\partial \mathrm{C}_{\mathrm{j}}}{\partial t}+\mathrm{C}_{\mathrm{j}} \frac{\partial v}{\partial z}+v \frac{\partial \mathrm{C}_{\mathrm{j}}}{\partial z}=\sum_{i} \nu_{j, i} r_{i}, \\
\mathrm{C}_{\mathrm{j}}(0, z)=\mathrm{C}_{\mathrm{j}, 0}(z), \quad \forall z \in[\text { in }, \text { out }] \\
\mathrm{C}_{\mathrm{j}}(t, 0)=\mathrm{C}_{\mathrm{j}, \text { in }}(t), \quad \forall t>0 \\
i=1, \ldots, 4, \\
j=1, \ldots, 7,
\end{array}
$$

where $j$ denotes the component, which could be $\mathrm{C}_{2} \mathrm{H}_{5} \mathrm{OH}, \mathrm{H}_{2} \mathrm{O}, \mathrm{CH}_{4}, \mathrm{CO}, \mathrm{CO}_{2}$, $\mathrm{CH}_{3} \mathrm{CHO}$ or $\mathrm{H}_{2}$ and $i$ denotes the reaction according to (1a)-(1d). Morover, $\mathrm{C}_{\mathrm{j}}$ is the concentration of the $j$-th component, $r_{i}$ is the reaction rate of reaction $i, v$ is the linear velocity of the gases, in is the set of inlet conditions while out is the set of outlet 
conditions, $\nu_{j, i}$ is the stoichiometric coefficient of component $j$ in reaction $i$ and $z$ is the axial position variable.

In order to solve the set of partial differential equations (PDEs) presented in (2a), $v$ and $r$ are expressed as functions of $\mathrm{C}_{\mathrm{j}}$. In the PFR with isobaric and isothermal conditions, it is assumed that $v$ varies with time and position within the reactor according to the following expressions [16]:

$$
\begin{aligned}
v & =v_{i n}(1+\varepsilon X), \\
X & =\frac{1-\frac{C_{j}}{C_{j} \text {,in }}}{1+\frac{C_{j}}{C_{j, \text { in }}}},
\end{aligned}
$$

where $X$ is the conversion rate of the ethanol while $\varepsilon$ is the molar relation for the considered reaction. Moreover, the volumetric flowrate $(Q)$ and molar flowrate of the different components $\left(F_{i}\right)$, which are assumed measured variables of the control problem, are computed as

$$
\begin{aligned}
Q & =A_{1} v, \\
F_{j} & =Q \mathrm{C}_{j},
\end{aligned}
$$

where $A_{1}$ is the section area of the tubular reactor. Reaction rates depend on concentration, temperature and pressure. According to [18], the following phenomenological kinetics have been considered for the four modeled reactions:

$$
\begin{aligned}
r_{1} & =k_{1}\left(P_{\mathrm{C}_{2} \mathrm{H}_{5} \mathrm{OH}}\right)^{m(P)}, \\
r_{2} & =k_{2} P_{\mathrm{C}_{2} \mathrm{H}_{5} \mathrm{OH}}, \\
r_{3} & =k_{3}\left(P_{\mathrm{CO}} P_{\mathrm{H}_{2} \mathrm{O}}-\frac{P_{\mathrm{CO}_{2}} P_{\mathrm{H}_{2}}}{k_{W G S}}\right), \\
r_{4} & =k_{4} P_{\mathrm{CH}_{3} \mathrm{CHO}} P_{\mathrm{H}_{2} \mathrm{O}}{ }^{3}, \\
k_{i} & =k_{\infty, i} \exp \left(-E_{a, i}\left(\frac{1}{R T}-\frac{1}{R T_{r e f}}\right)\right), \\
k_{W G S} & =\exp \left(\frac{4577.8}{T}-4.33\right), \\
m(P) & =1.2+0.23(P-4),
\end{aligned}
$$

where $k_{\infty, i}$ are the pre-exponential factors, $k_{i}$ the kinetic constants and $E_{a, i}$ the activation energies of each reaction. The reference temperature $T_{r e f}$ has been selected to be $873.15 \mathrm{~K}$, which is the highest temperature of the experiment. Besides, $m(P)$ is an exponential factor depending on pressure and $P_{\mathrm{C}_{2} \mathrm{H}_{5} \mathrm{OH}}, P_{\mathrm{CO}}, P_{\mathrm{H}_{2} \mathrm{O}}, P_{\mathrm{CO}_{2}}, P_{\mathrm{CH}_{3} \mathrm{CHO}}$ are the partial pressures of each component.

\subsection{Mass transfer of the membrane separation stage}

In this stage, the process of the hydrogen selective separation without chemical reactions takes place. A Pd-Ag metallic membrane is used to permeate only the hydrogen 
leaving the rest of the gas on the retentate side. The mass transfer mechanism can be expressed using the Sieverts' law as [4]

$$
\begin{gathered}
J_{H_{2}}=\frac{P_{e}}{\delta} A_{2}\left(\sqrt{P_{H_{2} \text { retentate }}}-\sqrt{P_{H_{2} \text {-permeate }}}\right), \\
\text { with } \quad P_{e}=P_{e 0} \cdot \exp \left(-\frac{E_{a}}{R T}\right),
\end{gathered}
$$

where $P_{e}$ corresponds with the gas permeability, $P_{e 0}$ is the pre-exponential factor, $R$ is the ideal gas constant, $T$ is temperature, $P_{H_{2} \text { retentate }}$ is the hydrogen partial pressure in the retentate side, $P_{H_{2} \text { permeate }}$ is the hydrogen partial pressure in the permeate side, $E_{a}$ is the apparent activation energy, $A_{2}$ is the surface area of the membrane and $J_{H_{2}}$ is the permeating hydrogen flux.

\subsection{Spatial discretization}

Each stage is divided into 20 slices of smaller size and each slice is considered as a continuous stirred tank reactor with homogeneous conditions. Backward finite differences are applied to transform the PDEs into ordinary differential equations (ODEs), which are expressed as

$$
\frac{\partial C_{j}}{\partial z} \cong \frac{C_{j}(z)-C_{j}(z-1)}{\triangle z} .
$$

\subsection{Parameters adjustment}

The model parameters have been adjusted to satisfy a set of static experimental data. Specifically, the molar flowrates of the different components $F_{i}$ were collected. Parameters of the reformer stage have been adjusted using experimental data obtained without the separation stage. Once these parameters have been fixed, the parameters of the separation stage have been adjusted using experimental data obtained with the whole (reformer stage plus separation stage) system. The pre-exponential factors and activation energies in (5) were adjusted in order that all the ethanol and acetaldehyde are completely transformed and the outlet molar flowrates of the rest of components are close to the experimental data. Specifically, the following steps were followed to fit the real data:

- First of all, adjust $k_{\infty, 2}$ and $E_{a, 2}$ to fit the quantity of $\mathrm{CH}_{4}$, which is only produced in reaction (1b)

- Then, adjust $k_{\infty, 1}$ and $E_{a, 1}$ to ensure that the whole conversion of ethanol is $100 \%$

- Then, adjust $k_{\infty, 4}$ and $E_{a, 4}$ to ensure that the conversion of acetaldehyde is $100 \%$

- Finally, adjust $k_{\infty, 3}$ and $E_{a, 3}$ according to the quantity of $\mathrm{H}_{2}$ from the experimental data. 
For each $k_{\infty, i}-E_{a, i}$ pair, $k_{\infty, i}$ was first adjusted at $T=T_{r e f}$ and then $E_{a, i}$ was adjusted using experimental data at different temperatures. The values of all the obtained parameters are shown in the Appendix. On the other hand, if $m(P)=1$, the kinetic model in (5) only fits for pressure at 1 bar. In order that it is suitable for pressures between $1 \mathrm{bar}$ to $14 \mathrm{bar}$, a power-law expression is required. Therefore, an exponential number $m(P)$ in $(5 \mathrm{a})$ was added. Equation $(5 \mathrm{~g})$ shows the relationship between the constant $m(P)$ and the pressure. The accuracy of the model with respect to the experimental data is assessed through the errors shown in Figure 3. It can be seen that the agreement between the experimental and simulated data is satisfactory. Finally, parameters $P_{e 0}$ and $E_{a}$ in (7) were also adjusted according to the real data of pure hydrogen obtained in the experiments. Exact values of both parameters are also given in the Appendix.

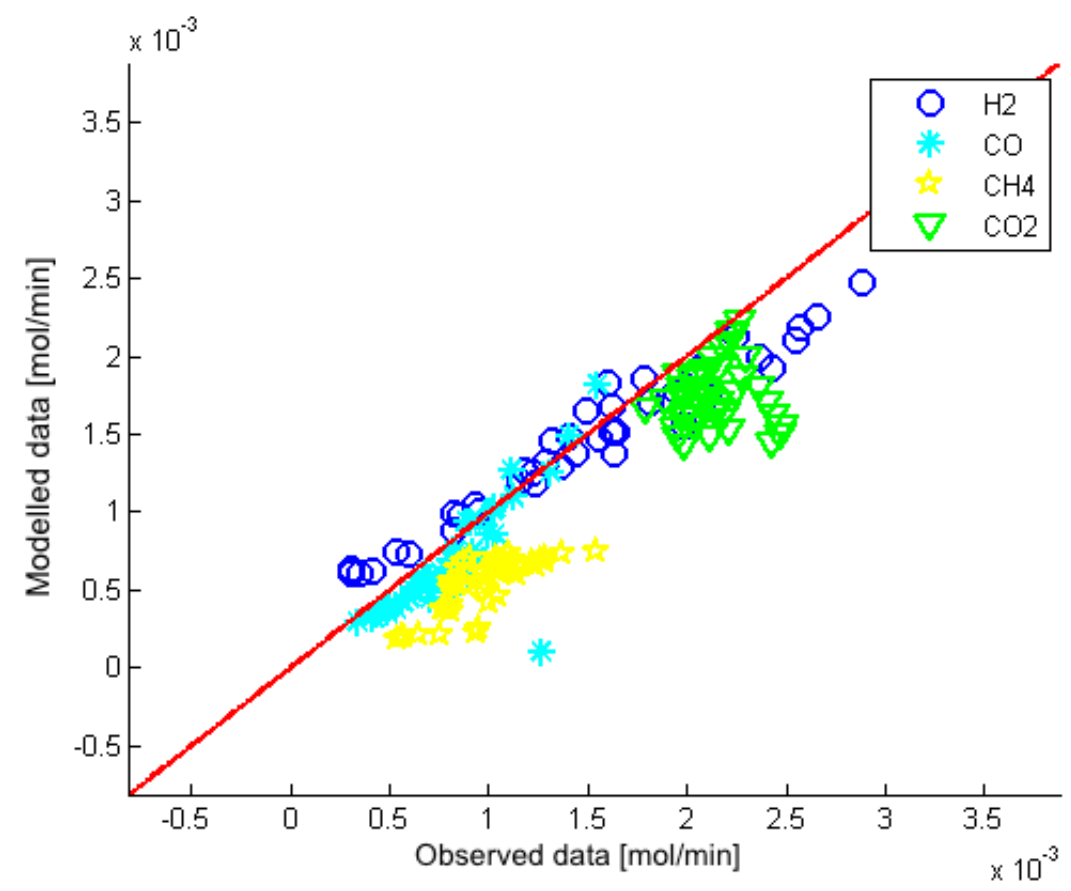

Figure 3: Model data versus experimental data. Red line shows a 1:1 correspondence

\subsection{Static analysis of the non-linear model}

The static and dynamic behaviour of the non-linear ESR model has been analysed in open loop. This information plays a significant role in the MPC controller design acting as a guidance for the manual tuning of the MPC controller.

The static results are obtained by solving the differential algebraic equations in (2) until all the derivatives with respect to time are equal to zero. Static flowrate profiles of each component with respect to the position in the axial direction of the reactor are shown in Figures 4(a) and 4(b) for the first and second stage of the reactor, respectively. 
The corresponding operating conditions are $P=8$ bar, $T=813.15 \mathrm{~K}$ and $S / C=3$. Some analysis based on the static profiles are included as following:

- In the first stage, ethanol is completely transformed into other products. Acetaldehyde firstly increases by the ethanol dehydrogenation and then decreases because it is further reformed with water to hydrogen and carbon dioxide. As can be seen in Figure 4(a), molar flowrates of hydrogen, carbon monoxide, methane and carbon dioxide are always increasing until the reaction finishes, while the molar flowrate of water decreases because of its participation into the reforming process. Ethanol has been completely consumed with $100 \%$ conversion, as is shown in Figure 4(c).

- In the second stage, at the retentate side, all flowrates are slightly reduced along the reactor. The reason for the flowrates decrease is the gases velocity decrease, necessary in order to keep a constant pressure at isothermal conditions. The only increasing flowrate in Figure 4(b) is the one of hydrogen at the permeate side because only hydrogen is capable of going through the membrane from the retentate to the permeate side.

\subsection{Dynamic analysis of the non-linear model}

The dynamic behaviour of the non-liner model is tested applying quick ramp changes to the inputs of the reformer. These changes are applied once the system is at equilibrium at the nominal conditions presented in the previous section. Figure 5 shows the molar outflow rates caused by ramps of $\pm 10 \%$ and $\pm 20 \%$ in ethanol, water and pressure inputs, respectively. In these figures, water and pure hydrogen outlet flowrates from the membrane separation stage are plotted. Through the observation of the output responses, some analyses are drawn as follows:

- Since a constant pressure is kept in the reactor, changes in the inlet flowrates are rapidly transmitted to the outlet flowrates.

- For the quick ramps in the ethanol input, hydrogen output presents a positive gain because more ethanol entering the reactor, more pure hydrogen can be obtained. However, the water has a negative gain because more water is required to participate into the reactions. Moreover, an inverse response can be seen in this output.

- For the quick ramps in the water input, it can be seen that less hydrogen is obtained with more water. The reason is that the partial pressure of hydrogen decreases, which results in decreasing permeability at the membrane separation stage.

- For the quick ramps in the pressure input, it can be seen that hydrogen has a positive gain and water has a negative gain. More hydrogen can be obtained working at higher pressures because the separation capability of the membrane increases almost linearly with the pressure at the retentate side. As a general 


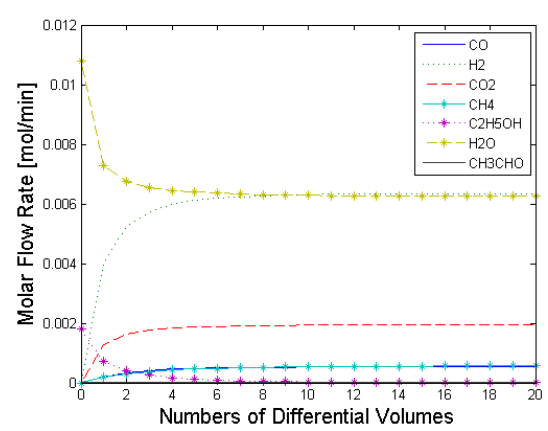

(a) Static profiles of the molar flowrate of each component in the reformer stage

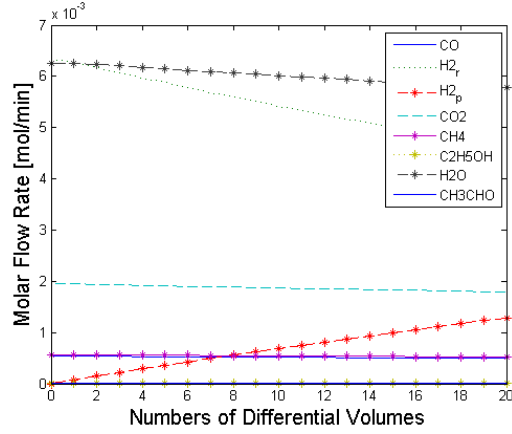

(b) Static profiles of the molar flowrate of each component in the membrane separation stage

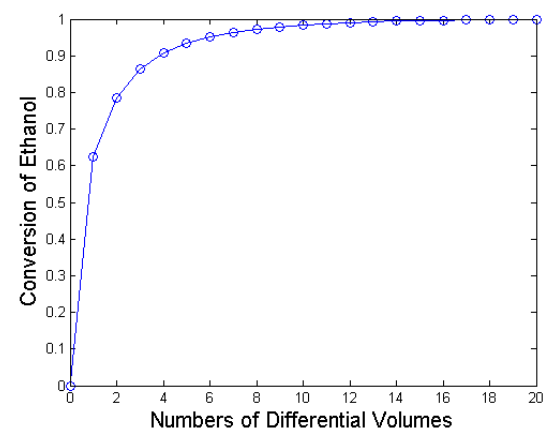

(c) Conversion rate of the ethanol

Figure 4: Static analysis of the ESR

conclusion, the separation stage has a strong influence on the flowrate of pure hydrogen.

\subsection{Control-oriented model}

In order to design the LMPC controller for the ESR with membrane separation, a linear control-oriented model is required. The selected nominal and linearization point is the one presented in Section 3.6. Three steps are followed to achieve this goal. Firstly, the linearisation of the non-linear model (NLsys) to get a linear system (Lsys) with 300 states. However, the Lsys model is a high order model and not fully-state observable, which is required for feedback control purposes. Then, the step of model-order reduction using Hankel Norm method is applied to obtain the linear-reduced model (LRsys) [20]. To this end, nine states are retained, all of them observable. Finally, since the nature of the MPC is discrete, a temporal discretization with a sampling time $T_{s}=0.5$ minutes is considered to obtain the linear-reduced discrete model (LRDsys). This is the control-oriented model used by the LMPC, which can be expressed in state-space 


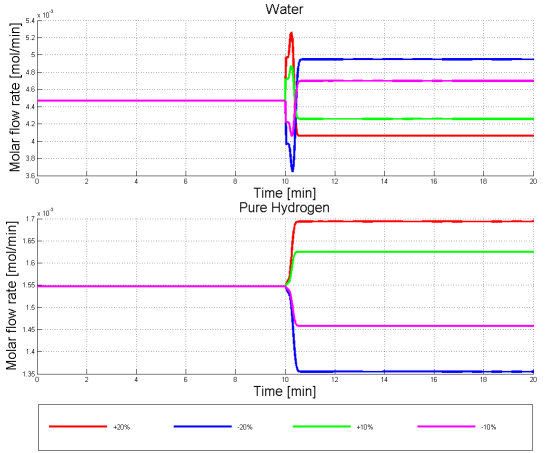

(a) Molar flowrates with $\pm 10 \%, \pm 20 \%$ quick ramp of the ethanol input
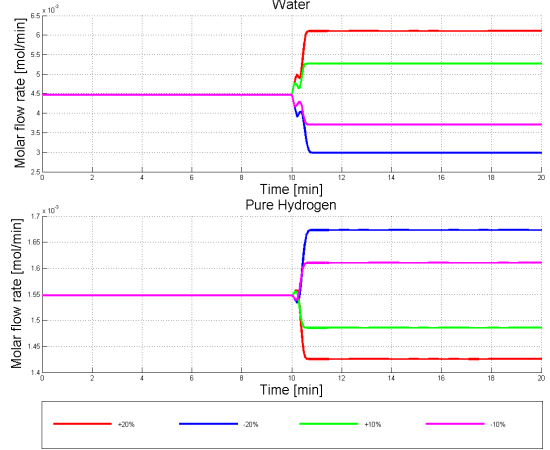

(b) Molar flowrates with $\pm 10 \%, \pm 20 \%$ quick ramp of the water input
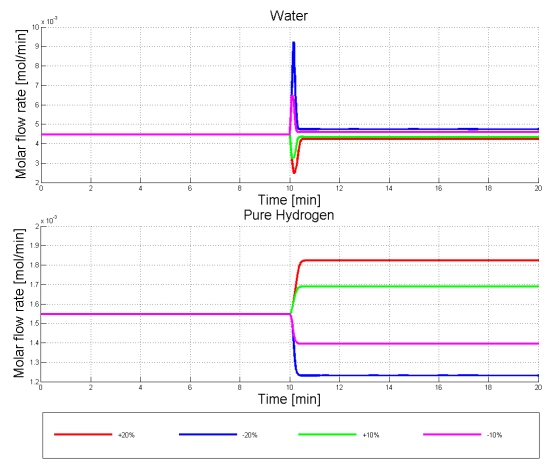

(c) Molar flowrates with $\pm 10 \%, \pm 20 \%$ quick ramp of the pressure input

Figure 5: Dynamic analysis of the ESR

form as

$$
\begin{aligned}
x_{r}(k+1) & =A_{r d} x_{r}(k)+B_{r d} u(k), \\
y(k) & =C_{r d} x_{r}(k)+D_{r d} u(k),
\end{aligned}
$$

where $k$ is the discrete time instant, $x_{r} \in \mathbb{R}^{9}$ are the state variables of the reduced model, $u \in \mathbb{R}^{4}$ are the manipulated inputs, which are the molar flowrate of ethanol, molar flowrate of water, pressure and temperature, respectively, and $y \in \mathbb{R}$ is the measured controlled output variable, which corresponds with the molar flowrate of pure hydrogen.

\subsection{Comparison between the nonlinear and linear models}

The nonlinear and linearised models are compared to verify their similarity and quantify the static errors. It is found that the steady state divergences between the lin- 
earised models and the non-linear model in the neighbourhood of the considered nominal working point are quite small so that they can be neglected. Moreover, there is no significant difference among the linear models. Thus, the error among the non-linear and linearised models comes from the linearisation rather than the model order reduction nor the time discretization. Table 1 presents the error percentage of the linear models with respect to the non-linear model. According to this table, the divergences of the hydrogen flowrate are limited to $2 \%$ when $\pm 10 \%$ and $\pm 20 \%$ changes from nominal conditions are applied to ethanol, water and pressure input flowrates.

Table 1: Percentage of hydrogen divergence comparing the linear and the non-linear models with ethanol, water and pressure inputs changes of $\pm 10 \%$ and $\pm 20 \%$ around the nominal steady state

\begin{tabular}{lcccc}
\hline Input & $+20 \%$ & $-20 \%$ & $+10 \%$ & $-10 \%$ \\
\hline Ethanol & $1.22 \%$ & $1.92 \%$ & $0.34 \%$ & $0.42 \%$ \\
Water & $0.21 \%$ & $-0.062 \%$ & $-0.043 \%$ & $-0.024 \%$ \\
Pressure & $0.99 \%$ & $1.78 \%$ & $0.28 \%$ & $0.37 \%$ \\
\hline
\end{tabular}

\section{The LMPC controller design}

\subsection{LMPC problem formulation}

According to the MPC methodology, four elements are required in order to design an MPC controller: the prediction model, the constraints on inputs and outputs, the cost function and the open-loop optimization problem with finite-horizon.

\subsubsection{Prediction Model}

For the ESR system, the prediction model corresponding to the LRDsys model in (9) involves the suitable iteration of the model over a prediction horizon and is expressed as $^{1}$

$$
\begin{aligned}
x_{r}(k+l+1 \mid k) & =A_{r d} x_{r}(k+l \mid k)+B_{r d} u(k+l \mid k), \\
y(k+l \mid k) & =C_{r d} x_{r}(k+l \mid k)+D_{r d} u(k+l \mid k),
\end{aligned}
$$

where $k$ is the discrete-time instant over the simulation time and $l \in\left[0, \ldots H_{P}-1\right]$ is the discrete-time instant over the prediction horizon $H_{p}$.

\footnotetext{
${ }^{1}$ Here, $z(k+l \mid k)$ denotes the prediction of the variable $z$ at time $k+l$ performed at time $\mathrm{k}$. For instance, $y(k+l \mid k)$ denotes the prediction of the system output.
} 


\subsubsection{Constraints}

The constraints of inputs and outputs are defined in the form of inequalities as

$$
\begin{aligned}
& u_{\min } \leq u(k+l \mid k) \leq u_{\max } \\
& y_{\min } \leq y(k+l \mid k) \leq y_{\max }
\end{aligned}
$$

where $u_{\min }$ and $u_{\max }$ are vectors of the lower and upper limits on the manipulated inputs, respectively while $y_{\min }$ and $y_{\max }$ are the lower and upper limits on the measured output. Considering the physical meaning of the inputs and outputs of the ESR, the constraint values must all be positive.

\subsubsection{Cost Function}

There are two control objectives in this paper. The first is aimed at tracking the set point of pure hydrogen flowrate while the second is to minimize the intake of ethanol. The cost function is the expression that collects the control objectives, which is written here as

$$
J(k)=\sum_{l=0}^{H_{p}-1}\left[\left\|e_{1}(k+l \mid k)\right\|_{M}^{2}+\left\|e_{2}(k+l \mid k)\right\|_{N}^{2}\right],
$$

subject to

$$
\begin{aligned}
& e_{1}(k+l \mid k)=y(k+l \mid k)-y_{r}(k \mid k), \\
& e_{2}(k+l \mid k)=u_{1}(k+l \mid k)-u_{1 \min }(k \mid k),
\end{aligned}
$$

where $y(k+l \mid k)$ is the sequence of predicted values of the controlled output (pure $\mathrm{H}_{2}$ flowrate) along $H_{p}, u_{1}(k+l \mid k)$ is the sequence of predicted ethanol inflows, $y_{r}$ is the reference profile to be tracked by the system output at each time instant and $u_{1 \min }$ corresponds to the minimal value allowed to the ethanol inflow. Besides, $M$ and $N$ are constant weighting matrices that reflect the prioritization of the control objectives collected in the multi-objective cost function (12), where $\|\cdot\|$ denotes de weighted Euclidean norm. Notice that the relation between $M$ and $N$ is given by two factors: the prioritization of the control objectives and the normalization of each term in the multi-objective cost function (12). In fact, each weight should be selected such that the value of each term belongs to the range $[0,1]$. Moreover, it is possible to weight the value of each term at every single time instant, fact that also enriches the functionality of both $M$ and $N$. Therefore, the relation $M>N$ is not straightforward then it is finally obtained by following an exhaustive trial-and-error procedure up to find a suitable trade-off such that the control objectives are properly reached.

\subsubsection{Optimization problem}

The formulation of the open-loop optimization problem adapted to the ESR with membrane separation can be written as 
Problem 4.1 (LMPC for ESR).

$$
\min _{u(k \mid k) \ldots u\left(k+H_{p}-1 \mid k\right)} J(k)
$$

subject to

$$
\begin{gathered}
x_{r}(k+l+1 \mid k)=A_{r d} x_{r}(k+l \mid k)+B_{r d} u(k+l \mid k), \\
y(k+l \mid k)=C_{r d} x_{r}(k+l \mid k)+D_{r d} u(k+l \mid k), \\
u_{\min } \leq u(k+l \mid k) \leq u_{\max }, \\
y_{\min } \leq y(k+l \mid k) \leq y_{\max }, \\
x_{r}(k \mid k)=\hat{x}_{r}(0 \mid k),
\end{gathered}
$$

where $\hat{x}_{r}(0 \mid k)$ is the initial vector of estimated states. According to the receding horizon strategy of the MPC controller, only the first component of the optimal sequence of control actions $u^{*}(k \mid k) \ldots u^{*}\left(k+H_{p}-1 \mid k\right)$ is applied to the process.

\subsection{Control specification}

Table 2 shows the specifications of the LMPC controller for the ESR with membrane separation. In this table, the nominal working point is determined and the constraints of the Manipulated Inputs (MI) and Measured Output (MO) are given as a percentage of variation around the corresponding nominal values. Notice that the temperature is an MI with constant value.

Table 2: Control specifications for the ESR

\begin{tabular}{lccc}
\hline Variables & Role & Working points & Constraints \\
\hline Ethanol inflow & MI & $1.8 \times 10^{-3}$ & $\pm 20 \%$ \\
Water inflow & MI & $10.8 \times 10^{-3}$ & $\pm 20 \%$ \\
Pressure & MI & 8 & $\pm 20 \%$ \\
Temperature & MI & 813.15 & 0 \\
Hydrogen outflow & MO & $1.547 \times 10^{-3}$ & $\pm 20 \%$ \\
\hline
\end{tabular}

\subsection{LMPC Closed-loop control}

Figure 6 shows the scheme of the LMPC closed-loop configuration. The LMPC controller computes the optimal control action at each sampling time based on Problem 4.1 and the first control action of the sequence is applied to the system. Besides, a full-order state observer is introduced in order to estimate the state vector from the measurement of the nonlinear system output. According to [28], a full-state Luenberger observer without disturbances has been designed, whose mathematical expression is written as

$$
\hat{x}_{r}(k+1)=\left(A_{r k}-L C_{r k}\right) \hat{x}_{r}(k)+B_{r k} u(k)+L\left(y(k)-D_{r k} u(k)\right),
$$

where $L$ is the estimator gain matrix that can be computed, as in the case of this paper, by using the MATLAB command dlqr. Once the states of the prediction model have been updated with the estimated states, the time horizons are slid and the optimization problem in 4.1 is repeated. 


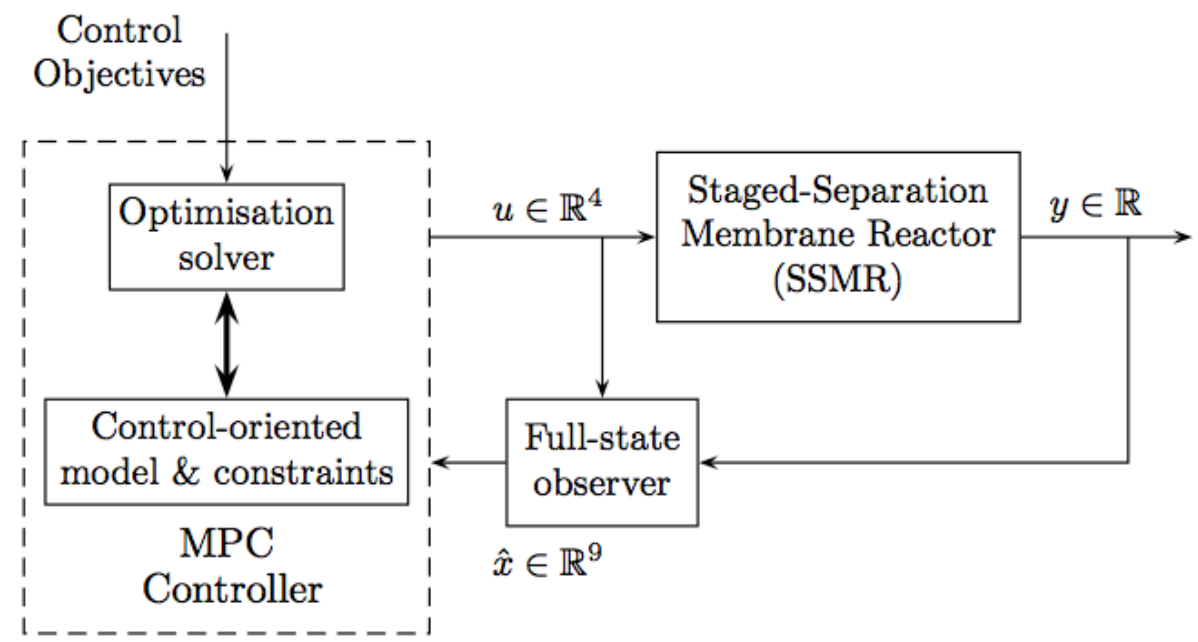

Figure 6: LMPC-based closed-loop configuration

\section{Simulation results}

Some parameters involved in the MPC controller can be tuned to meet the control objectives, which are the prediction horizon $\left(H_{P}\right)$, the control horizon $\left(H_{C}\right)$, and the weights for each control objective $(M$ and $N)$. In this work, $H_{C}$ has been given the same value as $H_{P}$. Eventually, $H_{P}=50$ (25 minutes), which ensures an adequate horizon for the closed-loop prediction. Regarding $M$ and $N$, two options are implemented in order that both control objectives can be weighted differently.

The first option is to assign the same weighs to $M$ and $N$ in order that both control objectives have the same priority. Figure 7 shows the results for this controller tuning case with setpoint changes of $\pm 10 \%$. In order to satisfy both control objectives at the same time, the controller searches the optimal inputs of water and pressure. However, it can be seen that, since ethanol minimization is given the same weight as hydrogen tracking, the hydrogen is always less than its reference within the simulation time (see Figure 7(b)). This shows that the capacity to meet both control objectives through the manipulation of water and pressure is limited. Therefore, if we consider that the principal control objective is to track the reference of hydrogen, the weighting option should be changed.

Considering the hydrogen flowrate setpoint tracking as the main objective, it is necessary to assign much more weight on $M$ than $N$. Figure 8 shows the simulation results related to this tuning case. In particular, Figure 8(a) shows the control actions applied to the real ESR system. The temperature is always maintained at its nominal value because it is not considered a manipulated input. Figure 8(b) shows the pure hydrogen flowrate and its reference. The set point is changed $\pm 10 \%$ at time 10 minutes, 25 minutes and 40 minutes. The pure hydrogen produced properly follows its reference. Furthermore, control actions change quite smoothly during the set point tracking in 
order to find the new steady state. Therefore, considering different dynamic and static aspects, it can be concluded that the control objective has been successfully achieved.

\section{Conclusions}

This paper presents an LMPC controller for the control of an ethanol steam reformer with membrane separation. The work is based on a non-linear distributed model whose parameters have been adjusted using experimental data. However, the LMPC controller is designed based on a linear, order reduced and discretised model that has been derived from the non-linear model. A comparison between the non-linear and linear models shows that the linear model is capable of representing the important dynamics of the ESR in a neighbourhood of the selected nominal operating point. Finally, the designed LMPC controller has been shown to be appropriate for setpoint tracking of hydrogen production for positive and negative changes up to $10 \%$. Also, the LMPC has shown its capability to optionally take into account the minimization of ethanol inflow through the tuning of some weighting parameters, although for physical reasons, the minimization of the ethanol inflow implies limitations in the achievable hydrogen production. Future work will address the analysis of the system at different operating conditions and the consideration of non linear MPC controllers. Also, it will address thermal model inclusion, uncertainty consideration as well as setup design improvement.

\section{Acknowledgement}

This paper has partially supported by the Spanish research project MICAPEM (ref. DPI2015-69286-C3-2-R, MINECO/FEDER). Mingming Li has been supported by the China Scholarship Council (CSC). Jordi Llorca is Serra Húnter Fellow and is grateful to ICREA Academia program.

\section{Acronyms}

\begin{tabular}{|c|c|}
\hline ATR & Auto-thermal Reforming \\
\hline ESR & Ethanol Steam Reformer \\
\hline HPLC & $\begin{array}{l}\text { High-performance Liquid } \\
\text { Chromatography }\end{array}$ \\
\hline LMPC & Linear Model Predictive Control \\
\hline LRDsys & Linear Reduced Discrete Model \\
\hline LRsys & Linear Model with Order Reduction \\
\hline Lsys & Linear Full-order Continuous Model \\
\hline MI & Manipulated Inputs \\
\hline MO & Measured Outputs \\
\hline MPC & Model Predictive Control \\
\hline MRs & Membrane Reactors \\
\hline NLsys & Non-linear Continuous Model \\
\hline
\end{tabular}




$\begin{array}{ll}\text { NMPC } & \text { Non-linear Model Predictive Control } \\ \text { ODEs } & \text { Ordinary Differential Equations } \\ \text { PDEs } & \text { Partial Differential Equations } \\ \text { PEMFC } & \begin{array}{l}\text { Polymer Electrolyte Membrane } \\ \text { Fuel Cell }\end{array} \\ \text { PFR } & \text { Plug Flow Reactor } \\ \text { PID } & \text { Proportion Integration Differentiation } \\ \text { POX } & \text { Partial Oxidation } \\ \text { ppm } & \text { Parts per million } \\ \text { PSA } & \text { Pressure-swing Adsorption } \\ \text { SR } & \text { Steam Reforming } \\ \text { WGS } & \text { Water Gas Shift }\end{array}$

\section{Nomenclature}

\begin{tabular}{|c|c|}
\hline$V_{1}$ & Volume, $\mathrm{m}^{3}$ \\
\hline$A_{1}$ & Section area of reactor, $\mathrm{m}^{2}$ \\
\hline$\varepsilon$ & Molar relation, dimensionless \\
\hline$V_{2}$ & Volume, $\mathrm{m}^{3}$ \\
\hline$\delta$ & Thickness of Pd-Ag membrane, m \\
\hline$A_{2}$ & Surface area of Pd-Ag membrane, $\mathrm{m}^{2}$ \\
\hline$D$ & Diameter of Pd-Ag membrane, $\mathrm{m}$ \\
\hline$R$ & Ideal gas constant, $\mathrm{J} \mathrm{mol}^{-1} \mathrm{~K}^{-1}$ \\
\hline$T$ & Temperature inside the reactor, $\mathrm{K}$ \\
\hline$T_{\text {ref }}$ & Temperature reference, $\mathrm{K}$ \\
\hline$v$ & Linear velocity of gases, $\mathrm{m} \mathrm{min}^{-1}$ \\
\hline$r$ & Reaction rates, $\mathrm{mol} \mathrm{m}{ }^{-3} \mathrm{~min}^{-1}$ \\
\hline$\nu$ & Stoichiometric coefficient, dimensionless \\
\hline$C$ & Concentration, $\mathrm{mol} / \mathrm{m}^{-3}$ \\
\hline$Q$ & Volumetric flow rate, $\mathrm{m}^{3} \mathrm{~min}^{-1}$ \\
\hline$F$ & Molar flow rate, $\mathrm{mol} \mathrm{min}^{-1}$ \\
\hline$P_{\mathrm{H}_{2} \_ \text {retentate }}$ & Hydrogen pressure in retentate side, $\mathrm{Pa}$ \\
\hline$P_{H_{2} \text { permeate }}$ & Hydrogen pressure in permeate side, $\mathrm{Pa}$ \\
\hline$P_{e}$ & Gas permeability, $\mathrm{mol} \mathrm{m}^{-1} \mathrm{~min}^{-1} \mathrm{~Pa}^{-0.5}$ \\
\hline$J_{H_{2}}$ & Flux of permeating hydrogen, $\operatorname{mol~min}{ }^{-1}$ \\
\hline$P$ & Pressure in the reformer stage, bar \\
\hline$P_{\mathrm{C}_{2} \mathrm{H}_{5} \mathrm{OH}}$ & Partial pressure of $\mathrm{C}_{2} \mathrm{H}_{5} \mathrm{OH}, \mathrm{Pa}$ \\
\hline$P_{\mathrm{H}_{2} \mathrm{O}}$ & Partial pressure of $\mathrm{H}_{2} \mathrm{O}, \mathrm{Pa}$ \\
\hline$P_{\mathrm{CO}}$ & Partial pressure of $\mathrm{CO}, \mathrm{Pa}$ \\
\hline$P_{\mathrm{CO}_{2}}$ & Partial pressure of $\mathrm{CO}_{2}, \mathrm{~Pa}$ \\
\hline$P_{\mathrm{CH}_{3} \mathrm{CHO}}$ & Partial pressure of $\mathrm{CH}_{3} \mathrm{CHO}, \mathrm{Pa}$ \\
\hline in & Reactor inlet \\
\hline out & Reactor outlet \\
\hline$T_{s}$ & Sampling time, minutes \\
\hline
\end{tabular}


$H_{P} \quad$ Prediction horizon

$M \quad$ Weighting matrix (control tuning)

$N \quad$ Weighting matrix (control tuning)

$u_{\text {min }} \quad$ Minimum values of manipulated inputs

$u_{\max } \quad$ Maximum values of manipulated inputs

$y_{\min } \quad$ Minimum values of measured outputs

$y_{\max } \quad$ Maximum values of measured outputs

\section{Appendix: Adjusted parameters}

\section{Reformer stage}

Activation energies of each reaction:

$E a_{1}=7.0 \times 10^{3} \mathrm{~J} \mathrm{~mol}^{-1}$

$E a_{2}=1.3 \times 10^{4} \mathrm{~J} \mathrm{~mol}^{-1}$

$E a_{3}=7.0 \times 10^{3} \mathrm{~J} \mathrm{~mol}^{-1}$

$E a_{4}=8.9 \times 10^{3} \mathrm{~J} \mathrm{~mol}^{-1}$

Pre-exponential factors of each reaction:

$k_{\infty 1}=5.025 \times 10^{3} \mathrm{~mol} \mathrm{~m}^{-3} \mathrm{~min}^{-1} \mathrm{bar}^{-1}$

$k_{\infty 2}=4.788 \times 10^{4} \mathrm{~mol} \mathrm{~m}^{-3} \mathrm{~min}^{-1}$ bar $^{-1}$

$k_{\infty 3}=4.548 \times 10^{3} \mathrm{~mol} \mathrm{~m}^{-3} \mathrm{~min}^{-1} \mathrm{bar}^{-2}$

$k_{\infty 4}=4.788 \times 10^{4} \mathrm{~mol} \mathrm{~m}^{-3} \mathrm{~min}^{-1} \mathrm{bar}^{-4}$

$\underline{\text { Membrane separation stage }}$

$E a=8.8 \times 10^{3} \quad \mathrm{~J} \mathrm{~mol}^{-1}$

$P_{e 0}=9.0 \times 10^{-7} \mathrm{~mol} \mathrm{~m}^{-3} \mathrm{~min}^{-1} \mathrm{~Pa}^{-0.5}$

\section{References}

\section{References}

[1] O. Z. Sharaf, M. F. Orhan, An overview of fuel cell technology: Fundamentals and applications, Renewable and Sustainable Energy Reviews 32 (0) (2014) 810_ 853. 
[2] D. George, P. Suresh, Detailed analysis of integrated steam ethanol reformer and high temperature polymer electrolyte membrane fuel cell, International Journal of Hydrogen Energy 41 (2) (2016) 1248-1258.

[3] E. McLeary, J. Jansen, F. Kapteijn, Zeolite based films, membranes and membrane reactors: Progress and prospects, Microporous and Mesoporous Materials 90 (1-3) (2006) 198-220.

[4] R. Koch, E. López, N. J. Divins, M. Allué, A. Jossen, J. Riera, J. Llorca, Ethanol catalytic membrane reformer for direct PEM FC feeding, International Journal of Hydrogen Energy 38 (14) (2013) 5605-5615.

[5] A. Li, C. J. Lim, J. R. Grace, Staged-separation membrane reactor for steam methane reforming, Chemical Engineering Journal 138 (1-3) (2008) 452-459.

[6] S. Tosti, M. Fabbricino, A. Moriani, G. Agatiello, C. Scudieri, F. Borgognoni, A. Santucci, Pressure effect in ethanol steam reforming via dense pd-based membranes, Journal of Membrane Science (2011) 65-74.

[7] A. Basile, A. Iulianelli, T. Longo, S. Liguori, M. De Falco, Pd-based selective membrane state-of-the-art, in: M. De Falco, L. Marrelli, G. Iaquaniello (Eds.), Membrane Reactors for Hydrogen Production Processes, Springer London, 2011, pp. 21-55.

[8] D. W. Juenker, M. van Swaay, C. E. Birchenall, On the Use of Palladium Diffusion Membranes for the Purification of Hydrogen, Review of Scientific Instruments 26 (1955) 888.

[9] A. Hedayati, O. Le Corre, B. Lacarriere, J. Llorca, Exergetic study of catalytic steam reforming of bio-ethanol over $\mathrm{Pd}-\mathrm{Rh} / \mathrm{CeO} 2$ with hydrogen purification in a membrane reactor, International Journal of Hydrogen Energy 40 (8) (2015) 35743581.

[10] M. Domínguez, G. Cristiano, E. López, J. Llorca, Ethanol steam reforming over cobalt talc in a plate microreactor, Chemical Engineering Journal 176-177 (0) (2011) 280-285.

[11] M. Domínguez, E. Taboada, E. Molins, J. Llorca, Ethanol steam reforming at very low temperature over cobalt talc in a membrane reactor, Catalysis Today 193 (1) (2012) 101-106.

[12] S. Losse, J. G. Vos, S. Rau, Catalytic hydrogen production at cobalt centres, Coordination Chemistry Reviews 254 (21) (2010) 2492-2504.

[13] J. D. Holladay, J. Hu, D. L. King, Y. Wang, An overview of hydrogen production technologies, Catalysis Today 139 (4) (2009) 244-260.

[14] M. Ni, D. Y. C. Leung, M. K. H. Leung, A review on reforming bio-ethanol for hydrogen production, International Journal of Hydrogen Energy 32 (15) (2007) 3238-3247. 
[15] E. Lopez, N. J. Divins, A. Anzola, S. Schbib, D. Borio, J. Llorca, Ethanol steam reforming for hydrogen generation over structured catalysts, International Journal of Hydrogen Energy 38 (11) (2013) 4418-4428.

[16] V. M. García, E. López, M. Serra, J. Llorca, Dynamic modeling of a threestage low-temperature ethanol reformer for fuel cell application, Journal of Power Sources 192 (1) (2009) $208-215$.

[17] E. López, N. J. Divins, J. Llorca, Hydrogen production from ethanol over Pd $\mathrm{Rh} / \mathrm{CeO}_{2}$ with a metallic membrane reactor, Catalysis Today 193 (1) (2012) 145150.

[18] I. Uriz, G. Arzamendi, E. López, J. Llorca, L. M. Gandía, Computational fluid dynamics simulation of ethanol steam reforming in catalytic wall microchannels, Chemical Engineering Journal 167 (3) (2011) 603-609.

[19] V. M. Garcia, M. Serra, J. Llorca, J. Riera, Design of linear controllers applied to an ethanol steam reformer for pem fuel cell applications, International Journal of Hydrogen Energy 38 (18) (2013) 7640-7646.

[20] D. Recio-Garrido, C. Ocampo-Martínez, M. Serra-Prat, Design of optimizationbased controllers applied to an ethanol steam reformer for hydrogen production, International Journal of Hydrogen Energy 37 (15) (2012) 11141-11156.

[21] J. M. Maciejowski, Predictive control with constraints, Prentice Hall, 2002.

[22] P. Rullo, L. N. Degliuomini, M. García, M. Basualdo, Model predictive control to ensure high quality hydrogen production for fuel cells, International Journal of Hydrogen Energy 39 (16) (2014) 8635-8649.

[23] R. Espinal, A. Anzola, E. Adrover, M. Roig, R. Chimentao, F. Medina, E. López, D. Borio, J. Llorca, Durable ethanol steam reforming in a catalytic membrane reactor at moderate temperature over cobalt hydrotalcite, International Journal of Hydrogen Energy 39 (21) (2014) 10902-10910.

[24] R. Espinal, E. Taboada, E. Molins, R. J. Chimentao, F. Medina, , J. Llorca, Cobalt hydrotalcite for the steam reforming of ethanol with scarce carbon production, RSC Advances 7 (2) (2012) 2946-2956.

[25] R. Espinal, E. Taboada, E. Molins, R. J. Chimentao, F. Medina, J. Llorca, Cobalt hydrotalcites as catalysts for bioethanol steam reforming: The promoting effect of potassium on catalyst activity and long-term stability, Applied Catalysis B: Environmental 127 (2012) 59-67.

[26] H. Li, A. Goldbach, W. Li, H. Xu, PdC formation in ultra-thin pd membranes during separation of $\mathrm{H}_{2} / \mathrm{CO}$ mixtures, Journal of Membrane Science 299 (1-2) (2007) 130-137.

[27] D. Wang, T. B. Flanagan, K. L. Shanahan, Permeation of hydrogen through preoxidized Pd membranes in the presence and absence of CO, Journal of Alloys and Compounds 372 (1-2) (2004) 158-164. 
[28] A. A. Stoorvogel, A. Saberi, B. M. Chen, Full and reduced-order observer-based controller design for $\mathrm{H}_{2}$ optimization, International Journal of Control 58 (4) (1993) 803-834. 

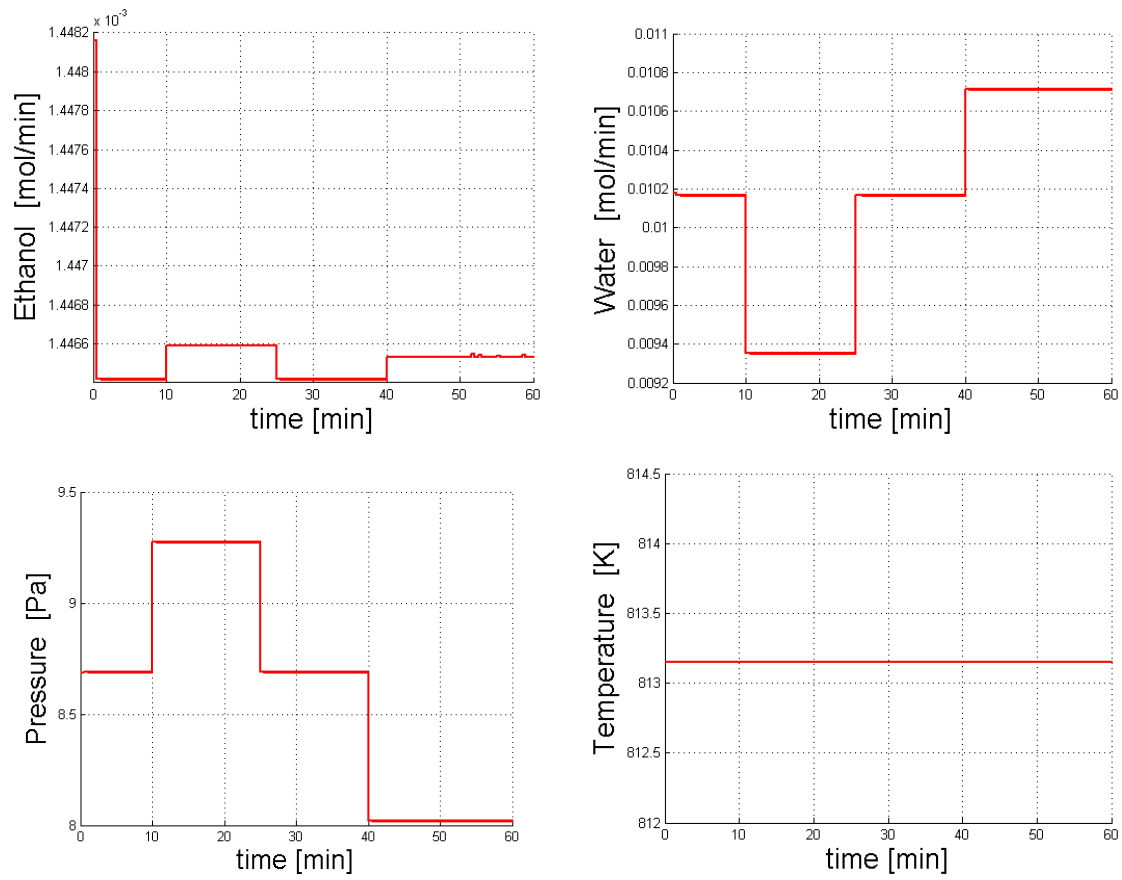

(a) Control actions of the closed-loop system with a positive reference

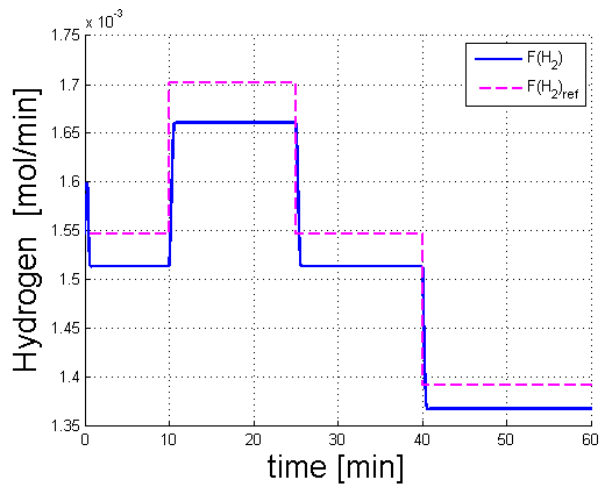

(b) Hydrogen output of the closed-loop system with a positive reference

Figure 7: Simulation results for the first case of controller tuning $(M=N)$ 

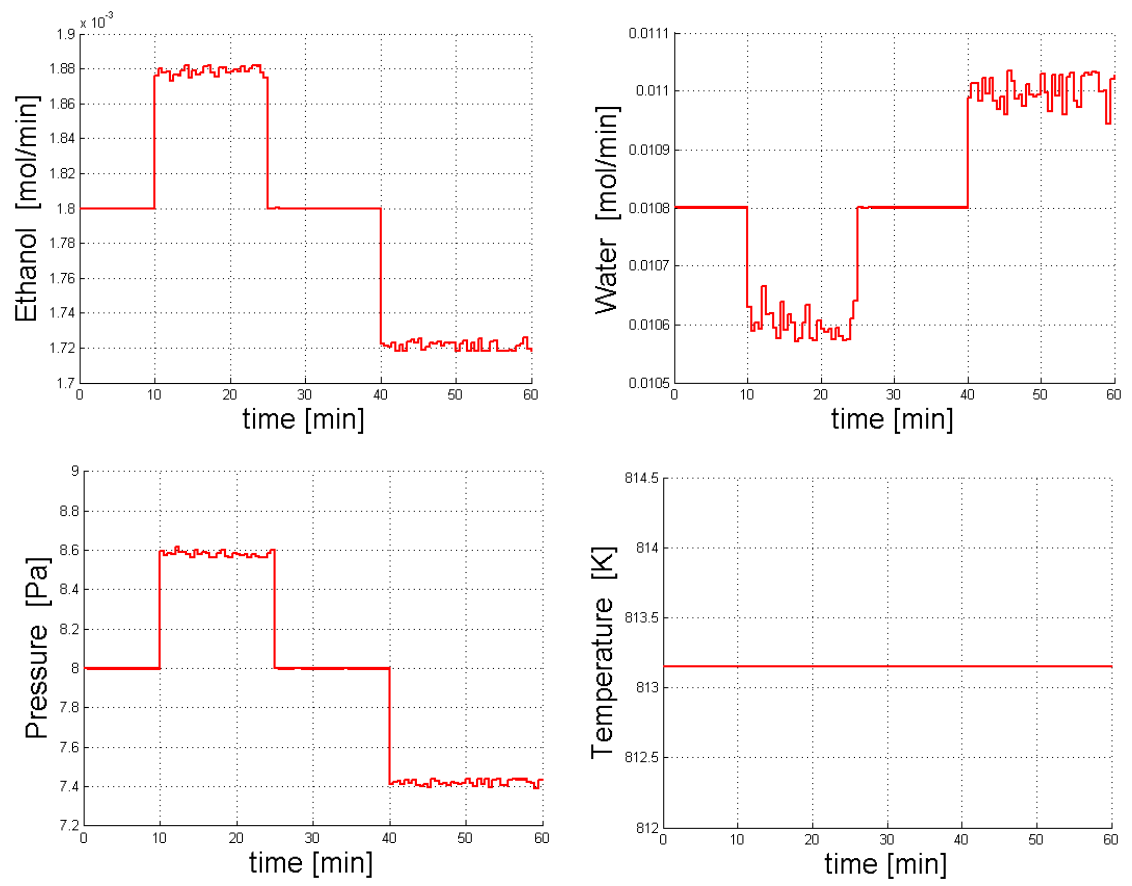

(a) Control actions of the closed-loop system with a positive reference

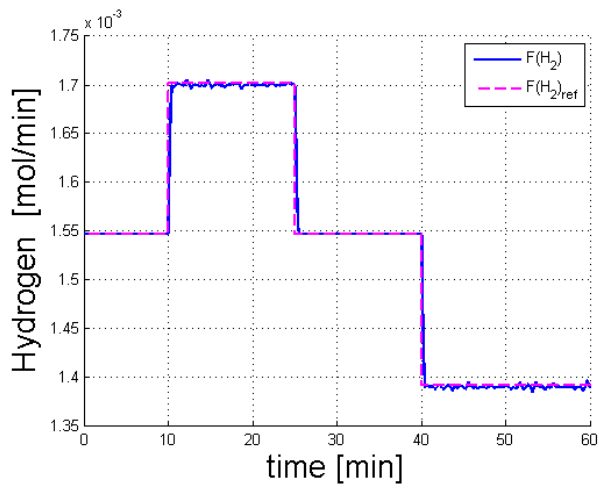

(b) Hydrogen output of the closed-loop system with a positive reference

Figure 8: Simulation results for the second case of controller tuning $(M>N)$ 